\title{
Relato de una experiencia: La Maranya, UNA ESCUELA DE DEMOCRACIA CIUDADANA CON JÓVENES
}

\section{Arecia Aguirre (UJI) - Mireia Beltrán — Iván Checa - Víctor Escoín - Virginia Morales - Noel Tomás \\ Equipo de Animadores Voluntarios del Centro Cívico La Maranya}

RESUMEN: El siguiente artículo busca difundir una experiencia asociativa enmarcada por la autogestión juvenil en el municipio de Benicàssim. Contamos cómo nace el centro cívico La Maranya y cuál es la ideología desde la que trabaja. La acción de esta asociación surge a partir del trabajo en el tiempo libre y de ocio como herramienta educativa. El centro parte de la participación cívica y democrática, el aprendizaje directo y el interés por los problemas de la sociedad para generar una escuela ciudadana de convivencia igualitaria. Este espacio trabaja con las jóvenes y los jóvenes como ciudadanas y ciudadanos activos conectados intergeneracionalmente con otros colectivos que lo conforman dentro de unos valores de progreso y participación social.

Palabras clave: jóvenes, asociacionismo, escuela ciudadana, democracia, tiempo libre.

RESUM: Aquest article pretén difondre una experiència associativa emmarcada per l'autogestió juvenil al municipi de Benicàssim. Expliquem el naixement del centre cívic La Maranya i quina és la ideologia des de la qual treballa. L'acció d'aquesta associació sorgeix arran del treball en el temps lliure i d'esplai com a eina educativa. El centre parteix de la participació cívica i democràtica, de l'aprenentatge directe $\mathrm{i}$ l'interès pels problemes de la societat per a generar una escola ciutadana de convivència igualitària. L'espai treballa amb les persones joves com 
a ciutadanes i ciutadans actius connectats intergeneracionalment amb altres col·lectius que el conformen dins de valors de progrés i de participació social.

Paraules clau: joves, associacionisme, escola ciutadana, democràcia, temps lliure.

ABSTRACT: The following article reports on an associational experience within the framework of young people's self-management in the town of Benicàssim. We describe how La Maranya Civic Centre came into being and the ideology it is grounded on. The association's action is based on using free time and leisure as an educational tool. The Civic Centre operates on the basis of democratic participation, non-formal learning and interest in society's problems to generate a citizens' school for egalitarian coexistence. The centre treats young people as active citizens who participate as equals with other age groups and associations, thus providing a context that fosters and promotes values associated with progress and democratic participation.

KEYWORDS: youth, associations, citizenship school, democracy, free time.

\section{Introducción}

Cuando hablamos de participación juvenil, nos preguntamos, citando a Palacios (2006), si los jóvenes no quieren participar o más bien no hemos sabido cómo hacerlo. Nuestro territorio no tiene articuladas unas políticas juveniles coherentes. Como mucho, podemos hablar de una red de infraestructuras municipales, más o menos idénticas, que la administración gestiona como lo haría con una oficina de turismo: mostrador, información y consumo. Sería interesante dar pasos hacia delante y superar el «ven, joven, a consumir lo que yo, la administración, te ofrezco» — tan definitorio de muchas políticas de juventud municipales - a un «vamos, joven, yo, la administración, me ofrezco para resolver problemas y cubrir necesidades». 
Hace falta enfrentarse a viejos retos, como el de fomentar la capacidad emprendedora de las personas jóvenes, sin olvidar, que emprender también es reivindicar. Hace falta dejar de organizarles actividades y montar proyectos serios que tengan como finalidad que aprendan a montar su propio ocio desde la solidaridad y el compromiso. Hace falta favorecer la participación juvenil en el desarrollo del municipio involucrando a las personas jóvenes en la transformación social de su propia realidad. Hace falta educar en el trabajo cooperativo, el diálogo, la reflexión, la diversidad y la conciencia crítica. En definitiva, hace falta promover la participación democrática de la ciudadanía.

Debido a este panorama social, suponemos importante la necesidad de compartir y conocer experiencias que dinamizan la participación juvenil desde valores de justicia social que dejan a un lado el paternalismo instalado en la sociedad para dar paso al trabajo asociativo desde la construcción comunitaria, desde la gestión independiente de las administraciones. Por ello, queremos dar a conocer, a lo largo de este artículo, un espacio construido sobre esta base. En el centro cívico La Maranya, situado en el municipio costero de Benicàssim, trabajamos a partir del asociacionismo juvenil autogestionado desde una educación popular, ciudadana e intergeneracional.

\section{Situándonos en el mapa: estructura y organización del centro cívico La Maranya}

Actualmente, La Maranya se encuentra ubicada en una de las plazas del casco antiguo del municipio de Benicàssim. Esta localidad pertenece al área metropolitana de Castellón, forma parte de la zona costera de esta provincia. El centro cívico La Maranya nace para agrupar, en una misma asociación, distintos colectivos generacionales. En este momento, un total de 107 personas somos socias del centro, de forma desglosada en los siguientes grupos: menores de 14 años, entre 14 y 30 años, mayores de 30 años, familias y algunas personas como colaboradoras puntuales. Todas estas personas socias nos agrupamos principalmente en tres grandes espacios como son el Esplai Cataflai (infantiles), la casa de juventud La Maranya (adoles- 
centes) y el Grup d'adults. Dentro de ellos encontramos diferentes grupos estables como son: el grupo de escalada, el grupo de cocina, el grupo de eurogames y rolgames, el grupo de guitarra, el grupo nómadas, el grupo de batucada y el grupo de consumo, entre otros.

Los inicios de esta asociación nos sitúan como casa de juventud La Maranya. Definimos casa de juventud, según la Federació Valenciana de Cases de Joventut (FVCJ), ${ }^{1}$ como «un servicio público, instrumento imprescindible para introducir en los jóvenes hábitos de participación, para generar en ellos consciencia de compromiso social, es decir, actitud activa y solidaria y ejercitar la capacidad de emprender. Es una pieza clave para la prevención de conductas y comportamientos antisociales al tiempo que es una herramienta para enfrentarse al problema de las drogas». En nuestro caso, La Maranya pertenecía hace unos años al Casal Jove municipal, dependiente del Ayuntamiento de Benicàssim. En aquel momento, los grupos promotores de la casa fueron el Esplai Cataflai, como grupo de jóvenes que trabaja la educación en el tiempo libre de niños y niñas; Estem Vius, grupo de teatro y activismo social; y Quatre gats, grupo de ocio nocturno alternativo. Después de una serie de problemas con la administración, se decidió asumir el distanciamiento con la administración pública, al pensar que era una oportunidad para actuar desde nuevos modelos de asociacionismo juvenil, autogestionados y sin vinculación partidista con agrupaciones políticas.

El nacimiento de La Maranya surge con el objetivo de seguir promoviendo la dinamización del ocio y tiempo libre de las personas jóvenes del municipio a través de proyectos educativos, laicos, democráticos y plurales desde el punto de vista político. En estos momentos nos enlazamos de forma más activa y directa con la FVCJ, sabiendo que actuando en soledad no podremos cambiar las cosas y que necesitamos compañeras y compañeros que nos ayuden en el día a día. Federarnos nos ayudó a ser más fuertes, a tener la parte legal y burocrática más controlada y a compartir el proyecto de transformación social con más jóvenes.

1. En la siguiente dirección se puede obtener más información sobre la Federació Valenciana de Cases de Joventut: http://lafederacio.org. 
Es a partir de este acontecimiento cuando La Maranya coge fuerza a través de la propia autogestión de las personas implicadas, del equipo de animadores y animadoras voluntarias y de gran parte del tejido de familias que, con anterioridad, ya participaba en el Casal Jove municipal. Se realizaron múltiples actividades, muchas de ellas propagadas a través de las redes sociales, que ayudaron a visibilizar la situación (campaña \#casaljovesensejoves, \#josocmaranya, videos como El portador de somnis...). Una de las acciones más sonadas, debido al número de seguidores y participantes en ella, fue el verkami: "Una casa per a La Maranya», ${ }^{2}$ promovido con el objetivo de conseguir fondos para poder alquilar un local donde establecer la Casa de Joventut. Conseguimos el objetivo, se alquiló un local y comenzó la andadura de obras, permisos, etc., que hacen que hoy La Maranya tenga su propia sede.

Hace casi un año la Casa de Joventut cambió su denominación a centro cívico con la finalidad de ampliar los colectivos de acción a infantil, adolescente y adulto. Aun así, el mayor espacio de actividad sigue siendo la Casa de Joventut y, por ello, se sigue apostando por la autogestión del centro por el colectivo adolescente como proceso de aprendizaje activo de esta agrupación. En este caso podríamos preguntarnos dónde se genera esa relación intergeneracional en la casa. Los espacios compartidos entre los tres colectivos son principalmente en tres lugares distintos, uno el Esplai, donde son las personas jóvenes quienes coordinan toda la actividad con las niñas y los niños y sus familias. Por otro lado, en las asambleas, en las que participamos todas las socias y socios del centro, mayores y menores de edad, para lanzar propuestas argumentadas a la casa. Además, se realizan actividades puntuales, como por ejemplo el último ciclo de debates (Recrea la teua ciutat: debats per a un nou projecte a Benicàssim), en el que está invitada a participar toda la juventud del centro, aunque es clave el trabajo del equipo de animadores y animadoras que colaboran en la coordinación del evento junto con otras personas adultas.

En La Maranya nos organizamos para hacer nuestro propio tiempo libre, cualquier persona puede apuntarse a los talleres o proponer actividades y organizarlas por ella misma. En el centro se facilitan los procesos de par-

2. Más información sobre el verkami en la siguiente dirección: http://www.verkami. com/projects/1445-una-casa-per-a-la-maranya. 
ticipación en función del proceso de maduración de la organización. Esto podemos apreciarlo a continuación al ver la pirámide de participación en la que nos basamos:

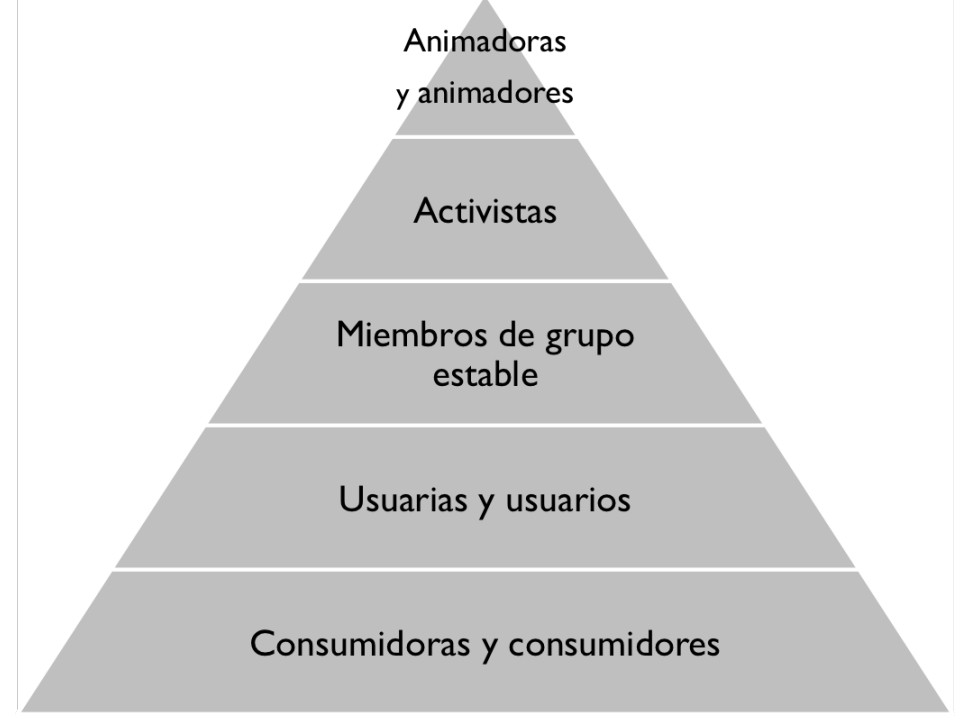

Figura 1. Pirámide de participación (Federació de Cases de Joventut)

1. Animadoras y animadores implicados en la dirección de las casas de juventud y el proyecto global del centro de manera consciente.

2. Activistas de grupos estables comprometidas con la dinamización de los colectivos. También son los miembros de la junta directiva de la Casa de Joventut.

3. Miembros de los grupos estables participantes en la actividad cotidiana del colectivo.

4. Usuarias y usuarios de cursos y servicios de la Casa de Joventut.

5. Consumidoras y consumidores de las actividades masivas organizadas por la Casa de Joventut.

En cuanto a la organización, podemos resumir en las siguientes líneas los puntos de encuentro y decisión más destacados: 
- El plenario mensual, donde representantes de los grupos estables y la persona representante de cada proyecto del centro nos reunimos para hacer una puesta en común de las acciones y tomar decisiones de modo argumentado sobre las mismas.

- La asamblea general, que realizamos dos veces al año, en octubre y en junio, y que definimos como el órgano soberano de la asociación. Está formada por todas las personas socias del centro y sus funciones principales son la elección de los órganos de dirección y la aprobación de la gestión y los planes de trabajo. Todas las personas participantes tienen derecho a realizar propuestas y acciones de forma argumentada en la asamblea, aunque actualmente el grupo con más responsabilidades sigue siendo el comprendido entre los 14 y 30 años, y son quienes únicamente tienen derecho a voto en las asambleas. Esta línea de trabajo se vincula directamente al objetivo de las casas de juventud de darles protagonismo directo en la gestión de sus propias iniciativas.

- Los grupos de tareas y la coordinadora apoyan a los activistas y las activistas que representan a cada grupo estable y a la persona animadora voluntaria como responsables en un ámbito concreto.

- La junta directiva, que es el órgano ejecutivo de las líneas generales, marcadas en la asamblea. Está formada por un o una representante de cada grupo estable o coordinadora de ámbito y por aquellos socios y socias que son escogidos por la asamblea general para llevar a cabo tareas de presidencia, secretaría y tesorería.

- El equipo de animadores y animadoras voluntarios (EAV). En él cada participante es responsable de una programación de intervención. El equipo de animadores y animadoras está compuesto por entre tres y diez personas que selecciona la junta directiva.

Hablemos ahora de la estructura orgánica de la asociación sustentada en este equipo, el cual lleva a cabo las programaciones de las distintas áreas de acción. Las áreas más comunes son: 
- Gestión interna.

- Publicidad y difusión.

- Programa de acción interna.

- Proyectos entre asociaciones.

- Creación y mantenimiento de grupos estables.

- Servicios, cursos y actividades puntuales.

- Programas en las áreas de cultura, deportiva, musical, social.

- Área de 14 a 17 años.

Uno de los pilares del trabajo en La Maranya se basa en que creemos que hace falta abrir puertas a la participación, sobre todo, para organizar actividades. Por ello en la casa no hay competencias entre actividades, cualquiera podemos proponer y organizar lo que queramos, siempre que respetemos los diferentes niveles establecidos y el ideario de la casa (lo veremos en el siguiente punto del artículo). El primer paso que debemos dar para proponer una actividad es muy sencillo, sólo se necesita que nos hagamos las siguientes preguntas:

- ¿De qué actividad se trata?

- ¿Por qué es interesante hacerla?

- ¿Cuándo será la actividad?

- ¿Cuánto cuesta?

- ¿Hace falta llevar alguna cosa?

- ¿Hace falta tener algo en cuenta?

Una vez nos cuestionamos la propuesta de este modo, podemos seleccionar el nivel de actividad que más se adecúe a la misma entre estos cuatro:

A. Actividades comunes, que se deciden en asamblea y las concreta el EAV.

B. Actividades de grupos estables que surgen de las activistas y tienen funcionamiento autónomo entre jóvenes. 
C. Talleres que se plantean por personas voluntarias con compromiso mínimo de un trimestre. Cualquier miembro puede proponer un taller y coordinarse con el EAV.

D. Propuestas puntuales.

En resumen, el centro cívico La Maranya se estructura y organiza según la política expuesta por la FCJV haciendo del espacio y del asociacionismo una escuela de democracia, práctica y activa, en la que los socios y las socias, sobre todo más jóvenes, gestionan su tiempo libre interactuando con otras agrupaciones con la finalidad de dinamizar nuestro compromiso ciudadano.

\section{Base ideológica del grupo: Manifiesto La Maranya}

A lo largo de estos cuatro años de andadura, nos hemos ido conformando en lo que actualmente es La Maranya. Un centro cívico en el que la diversidad intergeneracional de las personas que la conforman refuerza el proyecto asociativo. Nos declaramos como un centro cívico político:

Somos un espacio de debate, de crítica, donde hacer política tan necesaria en estos días para hacer de Benicàssim un lugar mejor para vivir. Nosotras no hacemos política de partidos, no somos un centro partidista porque defendemos la libertad de pensamiento, la conciencia crítica de las personas y el proceso de crecimiento de cada una. En un mundo gobernado por comerciantes y especuladores, apostamos por nuestra ciudadanía, la que lucha a golpe de sueños por ser antes persona que cosa. (Centro cívico La Maranya)

Para situar el proyecto de este centro cívico es fundamental conocer de cerca cuáles son nuestras manifestaciones ideológicas. Ellas representan la base del trabajo de cualquiera de los proyectos que puedan surgir en los grupos estables. Para conocer mejor el centro y su actividad veamos a continuación nuestro manifiesto: 


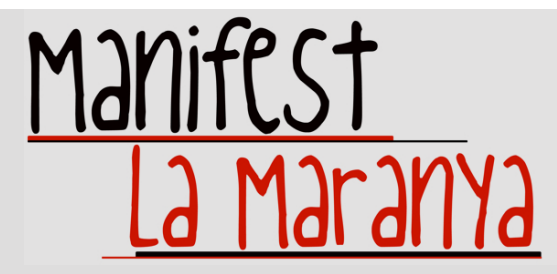

Somos una asociación que no pierde su sentido reivindicativo, porque no podemos cambiar las cosas si no lo intentamos. Somos conscientes de que nosotras solas no vamos a cambiar nada, ipero que nada cambiará sin nosotras!

- Solidaridad: queremos, y también tenemos obligación de ayudar a construir un mundo donde la paz y el respeto tengan mayor peso. Luchamos para disminuir las diferencias Norte-Sur, por un reparto justo de la riqueza.

- Igualdad: porque aquella égalité demandada en 1789 aún no es patente. Defendemos y trabajamos por la igualdad de los sexos, del acceso a los recursos, de oportunidades... Por una igualdad real.

- Laicidad: somos un centro laico, y eso quiere decir que puedes creer en lo que tú decidas. Incluso puedes ser atea y no creer en nada. A nosotras nos da igual, las creencias son algo privado.

- Contra el racismo y la xenofobia: nos gusta ser diferentes, por este motivo defendemos las minorías y trabajamos para una interculturalidad. Todas tenemos que aprender muchas cosas, porque todas hemos de enseñar muchas.

- Defensa de la cultura: defendemos una cultura diferente, una cultura libre, creada por nosotras mismas. Cultura de barrio, en pequeños grupos, no cultura de masas, ni cultura consumista, ni elitista. Trabajamos por una cultura que transforme, por una cultura liberadora.

- Democracia: la democracia es imperfecta, y eso todas lo sabemos. Pero es la única herramienta que tenemos para organizar la sociedad, hoy por hoy. Reclamamos el derecho a participar activamente de la vida social y política de nuestro pueblo, a usar los recursos públicos que la administración tiene la obligación de poner a nuestro alcance. Responsabilidad de hacerlo.

- Libertad: «Luchar por la libertad es ser libre ya» (Pierre Fougeyrollar). 
- Ecologismo: pensamos que el planeta no está en su mejor momento. Parece que podemos explotar indefinidamente. Defendemos el medio ambiente empezando por aquellos espacios que tenemos más cerca, cada árbol, cada parque...

- El rechazo al abuso del consumo de drogas: sabemos que mientras una joven se droga, un adulto con poder se frota las manos. Rechazamos cualquier peligro de adicciones que pueda limitar la libertad. Repudiamos el abuso de las drogas como entretenimiento para pasar el tiempo. Anhelamos la claridad, natural y creativa de las capacidades mentales y ponemos en marcha nuestros cimientos como proyecto alternativo al tiempo libre que fomente los hábitos culturales y deportivos.

- El rechazo a la violencia: rechazamos la violencia psíquica, física, de género, bullying... Pero sobre todo la violencia estructural, a menudo silenciosa, que provoca las injusticias sociales.

- Justicia social: todo derecho no universalizado no es un derecho, es un privilegio. Nuestro consumo afecta de manera determinante en la situación de otra gente en el planeta. «Si todos los indios tuvieran un coche aparcado en la puerta como hacemos los europeos, harían falta tres planetas para poder fabricarlos» (Galeano). Decimos no al consumismo, queremos un consumo crítico, un consumo responsable. Rechazamos la explotación infantil, rechazamos la pérdida de muchos derechos por nacer en lugares diferentes.

- Conciencia crítica: porque dudamos de que las cosas sean como nos las cuentan. Pensar críticamente es duro, pero tenemos que hacerlo antes de que otras decidan. No se pueden tomar decisiones sin reflexión. Reconocemos el pensamiento crítico como la única vía para mejorar la sociedad.

Como explicábamos antes, cualquier persona puede proponer actividad en La Maranya, pero sin eludir las ideas clave que constituyen este manifiesto y la estructura que sustenta a la casa, ya que son la base del proyecto educativo del centro. 


\section{Una escuela para la ciudadanía}

El cambio en la sociedad ya está ocurriendo: crisis, convulsiones, sufrimientos familiares, desmoronamiento de los sistemas económicos, paro juvenil máximo, aumento en las tasas universitarias, poco trabajo para gente sin experiencia... La población juvenil se ve abocada a una gran disponibilidad de tiempo libre que nadie, años atrás, les había enseñado a articular y a organizar. Es el momento de vivir el futuro, de nuevas ideas que antes nadie se arriesgaba a hacer. Ahora las actividades no han de consumirse, han de autoorganizarse. Para ello, debemos construir tejido. Es impensable que la juventud de los municipios viva encerrada en su grupo de amigos y amigas sin hacer red, sin conocer otras maneras de relación. Hemos visto cómo otros modelos de intervención juvenil más vinculados con la administración pública no han conseguido grandes efectos aparte del de lograr crear consumidores pasivos de servicios. Aun así, es importante valorar el trabajo de la animación juvenil llevado a cabo, pero sin olvidar que hacen falta nuevos modelos de intervención. La fuerza de la democracia es que la sociedad civil esté organizada asumiendo el papel de la resolución de los problemas que surgen y, por ello, debemos dinamizar que el compromiso ciudadano se concentre en acciones voluntarias a través de organizaciones no lucrativas.

Por todo esto, es necesario un modelo asociativo estructurado como en la experiencia de La Maranya, que dé protagonismo a la voz de la juventud y que ceda el poder de gobierno totalmente a la persona asociada. De este modo, conseguimos que la entidad pase a ser un verdadero laboratorio de ideas y una escuela de democracia donde se hacen de forma continua prácticas de lo que son las normas democráticas y justas de un sistema social que nos afecta directamente. El proyecto que hemos relatado responde a una manera de entender los procesos educativos desde la convivencia a través de contenidos, competencias, habilidades y valores en el día a día. Construye a partir de una base sustentada en la participación cívica y democrática, del aprendizaje directo y del interés por los problemas de la sociedad para generar una escuela ciudadana de convivencia igualitaria. Parémonos a pensar en la necesidad de avanzar en una toma de conciencia crítica de la ciudadanía de su propia realidad (Aguirre, 2012). 
Con los adolescentes, el tiempo libre puede convertirse en una gran oportunidad para trabajar hábitos de participación además de transmitirles valores de convivencia y sociabilidad. Para que esto sea posible, según Deltoro (2005), depende de cómo enfoquemos nuestro trabajo con adolescentes en el tiempo libre, si como una tarea educativa o como una simple planificación de entretenimientos. Para que esto no ocurra hay que potenciar el asociacionismo autónomo de la ciudadanía, porque queremos una sociedad autónoma y no una anclada en el paternalismo. No podemos tratar a la juventud como usuaria toda la vida. Algún día tendrá que tener la llave de las casas de juventud, si no, más vale cerrar esos espacios (Martí, 1998). La etapa juvenil se convierte en un momento clave para la formación dentro del concepto de ciudadanía, y si queremos que esta conceptualización esté basada en unos valores de justicia, de ética y de convivencia, hace falta actuar para conseguirlo (Aguirre, 2012). En este sentido, apostamos por el asociacionismo, ya que observamos la importancia de trabajar hacia una economía del bien grupal donde el apoyo mutuo, la empatía, la paz, el compañerismo, el diálogo, la democracia, el afecto, el respeto, la cooperación... sean valores que realmente constituyan el bienestar de las personas.

Montar infraestructuras paralelas a las necesidades e inquietudes de las personas y asumir que la ciudadanía es meramente consumista, contradice a Freire (1997) cuando nos habla de educar estando con el mundo y no de espaldas a él. Por todas estas razones vemos muy importante remarcar el papel que ha de desempeñar la administración pública con el objetivo de dinamizar a la ciudadanía. El ayuntamiento ha de promover la gestión de sus recursos y espacios por la propia juventud. Por un lado, debería facilitar recursos a las entidades juveniles de carácter tradicional para que puedan continuar manteniéndose y desarrollándose. Por otro lado, debe marcar una estrategia de apoyo claro a las nuevas iniciativas de carácter asociativo. Finalmente, ha de apostar por una formación de jóvenes dinamizadores con diferentes capacidades para trabajar y articular espacios sencillos dirigidos al aprendizaje de la autogestión en los municipios. Ésta es la única manera de conseguir de una vez por todas que la juventud, junto con otras personas jóvenes, adultas, incluso niños y niñas, desarrollen actitudes y valores que reviertan en una sociedad, sin duda, mejor que la actual. 


\section{Referencias}

Aguirre, A. (2012): «El diagnóstico social participativo juvenil como proceso de concientización: un estudio de caso», en Fernández, E. y Rueda, E. (coords.): La educación como elemento de transformación social. AUFOP-UVA-GEEP, Santander/Valladolid/Melilla.

Deltoro, E. (2006): Cómo trabajar con adolescentes en el tiempo libre, Certeza, Zaragoza.

Freire, P. (1997): A la sombra de este árbol, El Roure, Esplugues de Llobregat.

Palacios, J. L. (2006): «Los jóvenes no quieren participar». Ponencia presentada en los Encuentros de Tarazona, organizados por la FEMP (4, 5 y 6 de octubre). 